УДК 373+612.776.1

\title{
ЭКРАННОЕ ВРЕМЯ И ФИЗИЧЕСКАЯ РАБОТОСПОСОБНОСТЬ ПОДРОСТКОВ
}

\author{
Раевский Дмитрий Александрович \\ кандидат педагогических наук, доцент \\ «Государственный университет управления» \\ Чернова Мария Борисовна \\ кандидат педагогических наук, доцент \\ ФГБНУ «Институт возрастной физиологии \\ Российской академии образования» \\ Зайцева Галина Алексеевна \\ кандидат педагогических наук, доцент \\ Национальный исследовательский \\ технологический университет «МИСиС»
}

Аннотация: Обнаружена отрицательная взаимосвязь между суммарным экранным временем и физической активностью. В ряде работ показано, что чрезмерное увеличение экранного времени сопровождается уменьшением физической активности школьников в режиме дня и замедлением приростов показателей физической пригодности.

Цель исследования - выявить особенности аэробной и анаэробной работоспособности подростков, характеризующихся чрезмерно высокой среднесуточной величиной экранного времени.

Методология. В исследовании участвовали здоровые подростки 1315 лет. Экранное время определяли на основе хронометража и анкетного опроса подростков. Использовали комплекс тестов определения мышечной работоспособности и физической пригодности в широком диапазоне доступных нагрузок. Определяли также показатели физического развития и стадии полового созревания.

Результаты. Выявлены различия в уровне физической работоспособности подростков с низкими и высокими значениями экранного времени. Установлено, что у мальчиков, интенсивно применяющих цифровые технологии в режиме дня, ряд показателей анаэробной и аэробной 
работоспособности был меньше, чем у сверстников с низкими оценками экранного времени. Полученные результаты показывают, что подростки, использующие цифровые технологии более 4-х часов в сутки, характеризуются сниженной физической работоспособностью в различных зонах относительной мощности.

Заключение. Предполагается, что фактор чрезмерного экранного времени оказывает неблагоприятное влияние на физическую работоспособность подростков опосредованно, через снижение объема привычной физической активности в режиме дня. Сама же физическая активность снижается в следствие перераспределения бюджета свободного времени в сторону «избыточного» использования цифровых технологий.

Ключевые слова: Аэробная и анаэробная работоспособность, подростки, цифровые технологии, экранное время.

\title{
SCREEN TIME AND PHYSICAL FITNESS OF ADOLESCENTS
}

\section{Raevsky Dmitry Alexandrovich \\ Chernova Maria Borisovna Zaytseva Galina Alekseevna}

\begin{abstract}
A negative relationship was found between total screen time and physical activity. A number of studies have shown that excessive screen time is associated with a decrease in the physical activity of schoolchildren during the day and a slowdown in the growth of physical fitness parameters.

Study aim: To identify the peculiarities of aerobic and anaerobic performance of adolescents characterized by an excessively high average daily screen time.

Methods. The study included healthy 13-15-year-old adolescents. Screen time was determined on the basis of timing and a questionnaire for the adolescents. We used a set of tests for determining muscle performance and physical fitness in a wide range of available loads. Parameters of physical development and stage of puberty were also determined.
\end{abstract}

Results. We identified differences in the level of physical performance of adolescents with low and high screen time. It was found that boys who intensively use digital technologies during the day had lower anaerobic and aerobic 
performance parameters than their peers with low screen time estimates. The results obtained show that adolescents who use digital technologies for more than 4 hours a day have reduced physical performance in different relative power zones.

Conclusion. It is assumed that the factor of excessive screen time has an indirect adverse effect on the physical performance of adolescents through a decrease in habitual physical activity during the day. The physical activity itself decreases as a result of redistribution of free time towards the "excessive" use of digital technologies.

Key words: Aerobic and anaerobic performance, adolescents, digital technology, screen time.

Введение. Физическая работоспособность, как известно, во многом определяют уровень соматического здоровья и успешность адаптации школьников к воздействию широкого спектра природных и социальных факторов [1, 3 и др.]. Проблема оптимизации физической работоспособности и двигательной подготовленности учащихся приобретает особое значение в подростковом возрасте - критическом периоде онтогенеза, характеризующемся качественными преобразованиями физиологических и психологических функций, связанными с процессом полового созревания. Имеется немало научных данных, свидетельствующих о снижении уровня физической работоспособности и двигательной подготовленности современных школьников в процессе полового созревания. Наблюдаемая в последние десятилетия тенденция уменьшения двигательной активности и ухудшения физического состояния подростков [3] делает актуальными исследования, направленные на выявление особенностей их физической работоспособности в условиях современной информационно-насыщенной среды [13]. В ряде работ показано, что по мере увеличения экранного времени, время, отводимое на физическую активность, может сокращаться, а уровень физической пригодности - оставаться существенно сниженным $[9,16$, 4, 2 и др.]. Сходные результаты получены в исследованиях, использующих совокупные измерения экранного времени без учета специфики выполняемой деятельности, применяемых цифровых технологий и компьютерных средств. Обнаружена отрицательная взаимосвязь между суммарным экранным временем и физической активностью [16, 9, 18, 4, 7, 6 и др.]. Последнее может обусловливать замедление прироста физической работоспособности и уровня 
развития двигательных способностей в условиях чрезмерно активного использования средств цифровизации в повседневной жизни.

Цель исследования - выявить особенности аэробной и анаэробной работоспособности подростков, характеризующихся чрезмерно высокой среднесуточной величиной экранного времени.

Методология. В исследовании участвовали подростки 13-15 лет $(\mathrm{n}=168)$, отнесенные к основной медицинской группе для занятий физическими упражнениями.

Экранное время (ЭВ) определяли на основе хронометража и анкетного опроса подростков. Экранное время - это время, проведённое у экранов таких устройств как смартфон, планшет, компьютер, игровая приставка, ридер, телевизор [5, 8, 9, 7 и др.]. Рассчитывали экранное время по дням недели и суммарное экранное время за неделю. Данный показатель широко используется в современных исследованиях.

Анализ использования информационных технологий и компьютерных средств в режиме дня школьников проводится на основе опроса. Анкета для подростков включала следующие основные вопросы: с какого возраста и какими электронными устройствами пользуется дома и в школе; имеется ли доступ в Интернет; для чего использует различные электронные устройства; сколько времени проводит за электронными устройствами в течение дня; делает ли перерывы при использовании электронных устройств, какой деятельностью заполняет эти перерывы; использует электронные устройства во время еды и перед сном; контролируют ли родители контент и общее время использования электронных устройств и др. Величину компьютерной нагрузки (КН) определяли как суммарный показатель времени использования цифровых устройств в течение суток и недели.

Использовался комплекс тестов определения мышечной работоспособности и физической пригодности в широком диапазоне доступных нагрузок:

- зона максимальной мощности - максимальная анаэробная мощность (MАM) по тесту Маргариа, пиковая анаэробная мощность по Вингейтскому анаэробному тесту $\left(\mathrm{BAHT}_{5}\right)$, индекс накопления пульсового долга (ИНПД) после скоростного бега, мощность нагрузки, предельное время выполнения которой составляет 1 с (W1), прыжок в длину, бег 20 метров, подтягивания, максимальная сила; 
- зона субмаксимальной мощности - Вингейтский анаэробный тест $\left(\mathrm{BAнT}_{30}\right)$; время выполнения нагрузки $5 \mathrm{BT} / к г$ ( $\left.\mathrm{t} 5 \mathrm{BT} / к г\right)$, ИНПД после нагрузки 5 Вт/кг, мощность нагрузки, предельное время выполнения которой составляет 40 c (W40), поднимание туловища за 1 минуту из положения «лежа на спине»;

- зона большой мощности - время выполнения нагрузки 3 Вт/кг (t3Вт/кг), ИНПД после нагрузки 3 Вт/кг, показатель мощности нагрузки при пульсе 170 уд/мин (PWC170), максимальное потребление кислорода (МПК), мощность нагрузки, предельное время выполнения которой составляет $240 \mathrm{c}$ (W 240), шестиминутный бег;

- зона умеренной мощности - мощность нагрузки, предельное время выполнения которой составляет 900 c (W900), ватт-пульс (ВтП), коэффициент В уравнения Muller, характеризующий аэробную емкость, тест К.Купера.

Дозирование тестовой физической нагрузки осуществлялось с помощью велоэргометра. Определяли показатели физического развития (длину и вес тела, окружность грудной клетки, жизненную емкость легких и силу мышц спины и др.). Степень полового созревания оценивалась по методике, предложенной Tanner J.M. На её основе выделяли пять стадий полового созревания.

Статистическую обработку данных проводили с использованием пакета статистических программ. Сравнительный анализ включал расчет параметрических и непараметрических критериев статистической значимости различий.

Результаты. На основе полученных данных о среднесуточном времени использования компьютерных средств и цифровых технологий все подростки были проранжированы по величине экранного времени в порядке убывания. Для сравнительного анализа были отобраны испытуемые с самыми низкими $(\mathrm{n}=20)$ и самыми высокими $(\mathrm{n}=20)$ рангами. Экранное время у первой группы превышало 270 минут, а у второй - было меньше 55 минут. Как видно, подростки первой группы значительно превышали, рекомендуемый объем времени использования цифровых технологий в сутки [19, 17, 8 и др.].

Анализ полученных результатов выявил различия в уровне физической работоспособности у подростков с относительно низким и чрезмерно высоким экранным временем (рис.). 
Средние величины ряда показателей анаэробной алактатной работоспособности у мальчиков, интенсивно использующих цифровые технологии в режиме дня, были меньше, чем у сверстников с низким экранным временем. Различия ( $<<0,05-0,01)$ выявлены в отношение МАM, бега 20 метров. Показатели ВАнТ5, ИНПД 20м, Wmax, MC, результаты прыжка и подтягиваний статистически значимо не отличались. Несмотря на это проявилась тенденция снижения анаэробной гликолитической работоспособности, проявляемой в зоне максимальной мощности, с увеличением экранного времени.

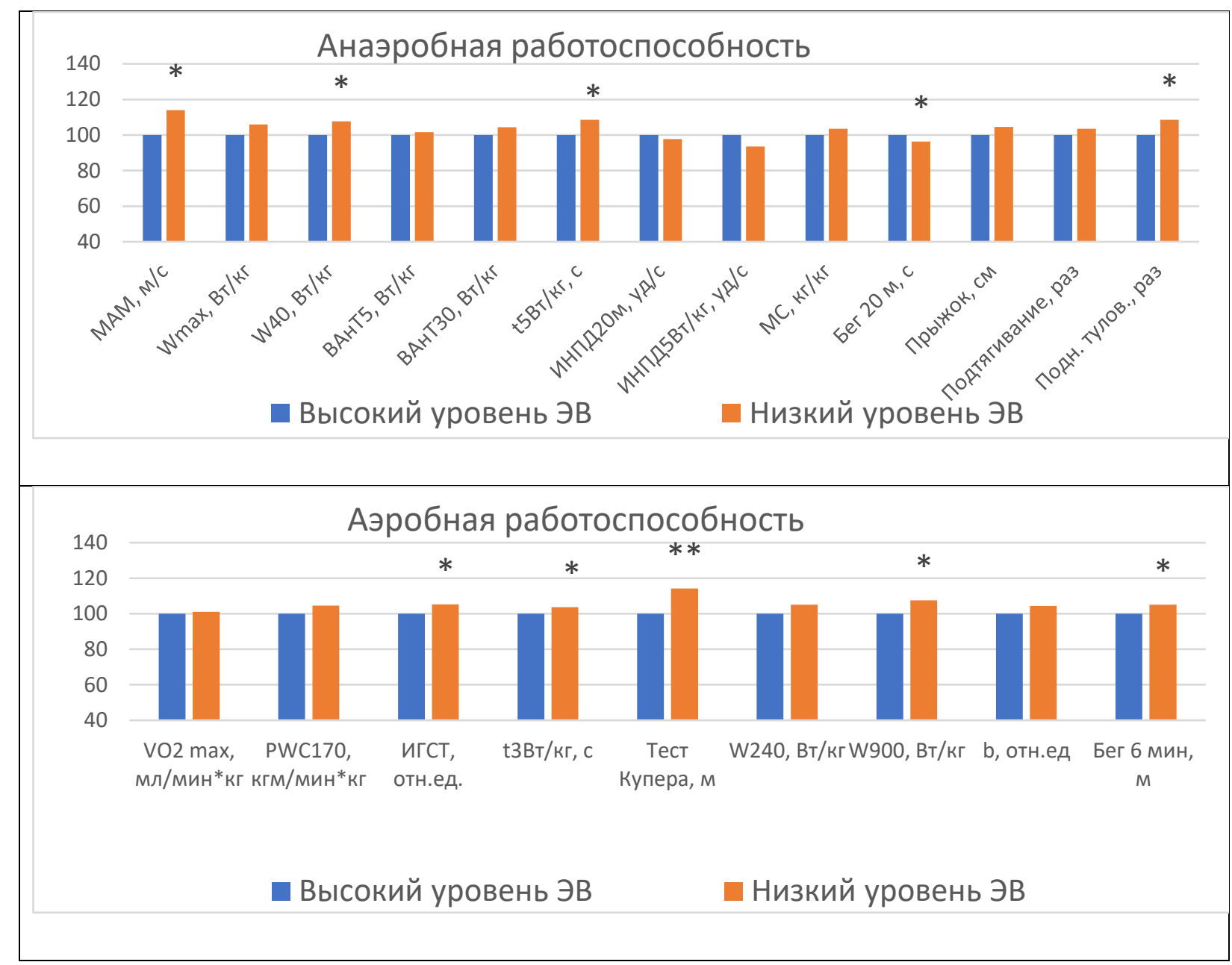

Рис. Аэробная и анаэробная работоспособность подростков 13-15лет с высоким и низким уровнем экранного времени

Примечание. За 100 \% приняты показатели в группе с высоким уровнем экранного времени (ЭВ). *,*, - статистически значимые межгрупповые различия при $\mathrm{p}<0,05$ и 0,01 соответственно. 
При сравнении показателей анаэробной гликолитической работоспособности обнаружены различия $(\mathrm{p}<0,05)$ в отношении $\mathrm{W} 40, \mathrm{t} 5 \mathrm{BT} / \kappa \Gamma$, теста поднимание туловища. Показатели ВАнТ 30 и ИНПД5Вт/кг у школьников с разным экранным временем не отличались. Однако и в этом случае, по мере увеличения экранного времени, наблюдалась слабая тенденция снижения показателей физической работоспособности, проявляемой в зоне субмаксимальной мощности.

Сопоставление показателей аэробной работоспособности, проявляемой в зонах большой и умеренной мощности, у подростков с высоким и низким экранным временем в ряде случаев выявило существенные ( $<<0,05-0,01)$ отличия. Эти отличия при работе в зоне большой мощности касались $\mathrm{t} 3 \mathrm{BT} / \mathrm{\kappa}$, ИГСТ, бега 6 минут, а при работе в зоне умеренной мощности - W 900 и теста Купера. В отношение же других показателей (VO2 max, PWC170, W240, коэф. b уравнения Мюллера) четко проявилась тенденция снижения физической работоспособности в широком диапазоне доступных нагрузок с увеличением экранного времени.

Сравнение физической работоспособности подростков с учетом уровня половой зрелости показало, что с увеличением стадии полового созревания отмечается существенный прирост $(\mathrm{p}<0,05-0,001)$ показателей анаэробной алактатной и анаэробной гликолитической работоспособности, тогда как аэробная работоспособность остается примерно на одном уровне. Динамика показателей физической работоспособности, выявленная на общей выборке подростков, проявилась и в группах школьников с высокими и низкими значениями экранного времени. Вместе с тем статистически значимые различия между подростками с разными стадиями полового созревания, связанные с интенсивностью использования цифровых технологий, не выявлены. Возможно, это обусловлено малым объемом сравниваемых выборок испытуемых с I, II, III, IV и V стадиями полового созревания.

Полученные результаты показывают, что подростки 13-15 лет, использующие компьютерные средства и цифровые технологии более 4-х часов 30-ти минут в сутки, характеризуются сниженной аэробной и анаэробной физической работоспособностью в различных зонах относительной мощности. Этот вывод в целом находит подтверждение в работах других авторов, изучавших взаимозависимости между интенсивностью использования детьми цифровых технологий в процессе 
жизнедеятельности и изменениями физической активности, двигательной подготовленности и функционального состояния организма $[16,9,4,7,6,2]$. В тех случаях, когда экранное время намного превышало рекомендованные оптимальные значения, обычно наблюдались надежные различия между детьми в отношении привычного объема физической активности, показателей развития двигательных способностей и заболеваемости $[14,19,5,11,18,15$, $12,8,10]$.

Для того, чтобы выявить влияние фактора «общее экранное время» на физическую работоспособность подростков с разными темпами полового созревания, необходимо дополнительно провести исследование с участием более представительной выборки испытуемых по каждой из 5-ти стадий полового созревания.

Заключение. Выявлены различия в уровне физической работоспособности подростков с низкими и избыточно высокими значениями экранного времени. Обнаружено, что у мальчиков, интенсивно применяющих цифровые технологии в режиме дня, ряд показателей анаэробной и аэробной работоспособности был меньше, чем у сверстников с низкими оценками экранного времени. В отношение же других показателей проявилась тенденция снижения физической работоспособности в широком диапазоне доступных нагрузок с увеличением экранного времени.

Полученные результаты показывают, что подростки, использующие цифровые технологии более 4-х часов 30-ти минут в сутки, характеризуются сниженной аэробной и анаэробной физической работоспособностью в различных зонах относительной мощности. Предполагается, что фактор чрезмерного экранного времени оказывает неблагоприятное влияние на физическую работоспособность подростков опосредованно, через снижение объема привычной физической активности в режиме дня. Сама же физическая активность снижается вследствие перераспределения бюджета свободного времени в сторону «избыточного» использования цифровых технологий. Работа поддержана РФФИ (грант № 20-013-00111a). 


\section{Список литературы}

1. Криволапчук, И.А. Особенности факторной структуры физической работоспособности мальчиков и девочек 9-10 лет/ И.А. Криволапчук, В.В. Мышьяков // Гигиена и санитария. - 2017. -Vol. 96. - №8. - С. 759-765.

2. Криволапчук, И.А. Функциональное развитие детей 6-7 лет с разным уровнем информатизации условий жизнедеятельности/ И.А. Криволапчук, М.Б. Чернова, А.А. Герасимова //Сибирский педагогический журнал. - 2020 №. 5. - C. 121-135.

3. Bull, F.C. World Health Organization 2020 guidelines on physical activity and sedentary behavior/ F.C. Bull, S.S. Al-Ansari, Biddle S., Borodulin K., Buman M.P., Cardon G., Carty C., Chaput J.P., Chastin S., Chou R., Dempsey P.C., DiPietro L., Ekelund U., Firth J., Friedenreich C.M., Garcia L., Gichu M., Jago R., Katzmarzyk P.T., Lambert E., Leitzmann M., Milton K., Ortega F.B., Ranasinghe C., Stamatakis E., Tiedemann A., Troiano R.P., van der Ploeg H.P., Wari V., Willumsen J.F. // Br J Sports Med. - 2020. - Vol. 54(24). - P. 1451-1462.

4. Cabanas-Sánchez, V. Associations of total sedentary time, screen time and non-screen sedentary time with adiposity and physical fitness in youth: the mediating effect of physical activity/ V. Cabanas-Sánchez., D. Martínez-Gómez, I. Esteban-Cornejo, A. Pérez-Bey, J. Castro Piñero, O.L. Veiga// J Sports Sci. - 2019. - Vol. 37. - № 8. - P. 839-849.

5. Domingues-Montanari, S. Clinical and psychological effects of excessive screen time on children / S. Domingues-Montanari// J Paediatr Child Health. 2017- Vol. 53(4). - P. 333-338.

6. Emm-Collison, L.G. Striking a Balance: Physical Activity, ScreenViewing and Homework during the Transition to Secondary School/ L.G. EmmCollison, S. Lewis, T. Reid, J. Matthews, S. J. Sebire, J. L. Thompson, R. Salway, R. Jago // Int J Environ Res Public Health. - 2019. - Vol. 16, №17. - P. 3174.

7. Ghekiere, A. Trends in sleeping difficulties among European adolescents: Are these associated with physical inactivity and excessive screen time?/ A. Ghekiere, J. Van Cauwenberg, A. Vandendriessche, J. Inchley, M. Gaspar de Matos, A. Borraccino, I. Gobina, J. Tynjälä, B. Deforche, B. T. De Clercq //Int J Public Health. - 2019. - Vol. 64(4). - P. 487-498. 
8. Guerrero, M.D Screen time and problem behaviors in children: exploring the mediating role of sleep duration / M.D. Guerrero, J.D. Barnes, M.S. J.P. Chaput, M.S. Tremblay //Int J Behav Nutr Phys Act. - 2019. - Vol. 16. - P. 105-109.

9. Hardy, L.L. Association Between Sitting, Screen Time, Fitness Domains, and Fundamental Motor Skills in Children Aged 5-16 Years: Cross-Sectional Population Study/ L.L. Hardy, D. Ding, L.R. Peralta, S. Mihrshahi, D. Merom //, J Phys Act Health. - 2018. - Vol. 15. - № 12. - P. 933-940.

10. Hinterlong, J.E. Association of multimedia teaching with myopia: A national study of schoolchildren / J.E. Hinterlong, V.L. Holton, C.C. Chiang, C.Y. Tsai, Y.M. Liou // J Adv Nurs. - 2019. - Vol.75(12). - P. 3643-3653.

11. Hoge, E. Digital Media, Anxiety, and Depression in Children / E. Hoge, D. Bickham, J. Cantor/ E. Hoge, D. Bickham, J. Cantor // Pediatrics. - 2017. - Vol. 140 (Suppl 2). - P.76-S.80.

12. Janssen, X. Associations of screen time, sedentary time and physical activity with sleep in under 5s: A systematic review and meta-analysis / X. Janssen, A. Martin, A.R. Hughes, C.M. Hill, G. Kotronoulas, K. Hesketh // Sleep Med Rev. - 2019. - Vol. 49. - P. 101-226.

13. Krivolapchuk, I. Modified Informatization Index of Children's Life/ I. Krivolapchuk, M. Chernova, A. Gerasimova, V. Chicherin, V. Myshyakov // In: Popkova E.G., Sergi B.S. (eds) "Smart Technologies" for Society, State and Economy. ISC 2020. Lecture Notes in Networks and Systems, 2021. - Vol. 155. P. 548-557.

14. LeBlanc, A.G. ISCOLE Research Group. Correlates of Total Sedentary Time and Screen Time in 9-11 Year-Old Children around the World: The International Study of Childhood Obesity, Lifestyle and the Environment/ A.G. LeBlanc, P.T. Katzmarzyk, T.V. Barreira, S.T. Broyles // PLoS One. - 2015. - Vol. 10(6):e0129622.

15. Mustafaoğlu, R. The negative effects of digital technology usage on children's development and health. Addicta: The Turkish / R. Mustafaoğlu, E. Zirek, Z. Yasacı, A. Razak Özdinçler // Journal on Addictions. - 2018. - № 5. - P. 227-247.

16. Potter, M. Behavior Tracking and 3-Year Longitudinal Associations Between Physical Activity, Screen Time, and Fitness Among Young Children /M. Potter, J.C. Spence, N. Boulé, J.A. Stearns, V. Carson // Pediatr Exerc Sci. - 2018. - Vol. 30. - № 1. - P. 132-141. 
17. Saunders, T.J. Screen Time and Health Indicators Among Children and Youth: Current Evidence, Limitations and Future Directions / T.J. Saunders, J.K. Vallance // Appl Health Econ Health Policy. - 2017. - Vol. 15(3). - P. 323-331. '

18. Straker, L. Conflicting Guidelines on Young Children's Screen Time and Use of Digital Technology Create Policy and Practice Dilemmas/ L. Straker, J. Zabatiero, S. Danby, K. Thorpe // J Pediatr. - 2018. - Vol. 202. - P. 300-303.

19. Tremblay, M.S. Canadian 24-hour movement guidelines for children and youth: an integration of physical activity, sedentary behaviour, and sleep / M.S. Tremblay, V. Carson, J-P. Chaput, S. Connor Gorber, T. Dinh, M. Duggan // Appl Physiol Nutr Metab. - 2016. - Vol. 41(6 Suppl 3). - P. 311-327.

20. Vieira, J.A. Effectiveness of a multicomponent intervention on the screen time of Brazilian/ J.A. Vieira, L. R. de Lima, D. A. Silva, E. L. Petroski // Motriz, Rio Claro. - 2018. - Vol.24, Issue 3. - e0046-18. 